\title{
Utilizzo degli antibiotici: risultati di un sondaggio condotto su un campione di dentisti italiani
}

\section{The use of antibiotics: data analysis of a survey among a sample of italian dentists}

\author{
Ricevuto il 9 luglio 2017 \\ Accettato il 15 gennaio 2018 \\ *Autore di riferimento \\ Marco Giargia \\ drm.studiogiargia@gmail.com
}

\author{
${ }^{1}$ Libero professionista in Albisola (SV) \\ ¿Università di Brescia, Clinica \\ odontoiatrica, Dipartimento di specialità \\ chirurgiche, scienze radiologiche \\ e sanità pubblica
}

\section{ABSTRACT}

\section{RIASSUNTO}

\section{OBIETTIVI}

Valutare il ricorso all'impiego degli antibiotici sistemici e locali nel trattamento delle patologie parodontali e peri-implantari in un gruppo di dentisti italiani, nonché la coerenza con le indicazioni scientifiche e il problema dell'antibiotico resistenza.

\section{MATERIALI E METODI}

I partecipanti sono stati reclutati tramite e-mail e invitati a partecipare a un sondaggio on-line senza alcun criterio di selezione.

\section{RISULTATI}

Hanno partecipato al sondaggio 1079 dentisti. L'impiego di antibiotico sistemico per le patologie parodontali e peri-implantari risulta molto frequente. Amoxicillina e acido clavulanico i farmaci più usati. Diffuso anche l'uso di prodotti locali, inclusa la clorexidina.

\section{CONCLUSIONI}

II ricorso ad antibiotici per via sistemica risulta l'approccio più frequente anche in assenza di indicazioni stringenti. Antibiotici e disinfettanti applicati localmente sono molto utilizzati anche per quadri clinici fortemente differenti. Frequente è anche il ricorso a sostanze non idonee per lo scopo o utilizzate in modo improprio ma economiche (clorexidina).

\section{PAROLE CHIAVE}

\section{Antibiotico sistemico}

Antibiotico topico

- Ascesso parodontale

- Peri-implantiti resistenze batteriche

\section{RESULTS}

\section{OBJECTIVES}

To evaluate the use of systemicalIy and locally delivered antibiotics for the treatment of periodontal end peri-implant diseases in a sample of italian dentists as well as the accordance to evidence based indications and the issue of antibiotic resistance.

\section{MATERIALS AND METHODS}

Participants were invited by e-mail to join the survey by answering to an on-line questionnaire without any selection criteria.

1079 dentist joined the survey. The use of systemic antibiotics for periodontal and peri-implant disease was highly frequent. Amoxicillin+clavulanic acid were the most prescribed. The use of local drugs including clorexydine was also very frequent.

\section{CONCLUSIONS}

Systemic administration of antibiotics appears as a first choice treatment also when not strictly indicated. Locally delivered antibiotics and antiseptic are also very frequently used in conjunction with different altered clinical conditions. Products which are inadequate for the purpose or inappropriately applicated but cheap are often used (clorexydine).

\section{KEY WORDS}

Systemic antibiotics

- Bacterial resistance

- Topical antibiotics

- Periodontal abscess

- Peri-implantitis 


\section{INTRODUZIONE}

La scoperta dei primi antibiotici e la successiva sintesi di nuove molecole ha rappresentato un enorme passo avanti per l'umanità, consentendo di curare patologie fatali, prevenire epidemie e salvare numerose vite umane.

Nel tempo, la realizzazione di nuove formulazioni farmaceutiche sistemiche e la maneggevolezza dei farmaci ne ha ulteriormente diffuso l'uso e i benefici.

All'ampia diffusione, però, ha fatto seguito il problema dell'insorgenza di ceppi batterici resistenti ai farmaci stessi con conseguente perdita o diminuzione della loro efficacia e una recrudescenza di patologie, soprattutto respiratorie, che si pensavano ormai controllabili.

Uno stipite batterico è definito resistente a un farmaco quando è in grado di moltiplicarsi in presenza di concentrazioni dello stesso capaci di impedire la moltiplicazione della maggior parte degli altri batteri. II problema risiede, ovviamente, nella cosiddetta "resistenza acquisita" da cui dipende la perdita di efficacia imprevedibile del farmaco conseguente a mutazioni spontanee e non tanto in quella "naturale o intrinseca", nota a priori[1].

La possibilità di selezionare queste mutazioni spontanee e casuali e, quindi, ceppi resistenti, è strettamente legata alla frequenza di somministrazione e va posta in relazione anche alle abitudini prescrittive della popolazione medica.

Oggi, le dimensioni planetarie del fenomeno e la mancata auspicabile inversione di tendenza hanno portato alla necessità di interventi di sensibilizzazione da parte delle organizzazioni internazionali.

L'European Center for Disease Control and Prevention (ECDC) nel 2015 ha dichiarato, infatti, che il consumo di anti- biotici in Europa non diminuisce. L'Italia, nel 2014, con 27,8 dosi/giorno in prescrizione non ospedaliera, si colloca al quinto posto in Europa (media UE di 21,6 dosi/giorno).

Molti autori, a livello internazionale, hanno intrapreso studi per valutare la modalità e le consuetudini prescrittive nella popolazione medica, evidenziando come la categoria odontoiatrica contribuisse per valori che oscillavano tra il 6,5\% e l' $11 \%$ del totale delle prescrizioni[2].

Fawziah Marra, in uno studio condotto nella Columbia Britannica (Canada), riscontrava che i dentisti rappresentavano la seconda classe di figure sanitarie che più prescriveva antibiotici sistemici (11,3\% su 2,6 milioni di prescrizioni nel 2013) con una crescita progressiva nel periodo 1996-2003 [3]

Secondo Pipalova et al., in Repubblica Ceca i dentisti erano stati responsabili del $6,5 \%$ del totale delle prescrizioni di antibiotici nel 2006 e dell'8,5\% nel $2012^{[4]}$. In Galles, nel 2008 il 9\% delle prescrizioni sono state attribuite agli odontoiatri. Gli antibiotici più consigliati erano la penicillina e il metronidazolo[ ${ }^{[5]}$. In Spagna, Poveda-Roda et al. hanno stimato che il $10 \%$ della totalità delle prescrizioni di antibiotico era indirizzato al trattamento delle infezioni odontogene ${ }^{[6]}$.

Mainjot et al., in Belgio, hanno chiesto ai dentisti di annotare ogni prescrizione di antibiotico durante due settimane lavorative. Su 24.421 pazienti visitati, a 1033 venne prescritto un antibiotico con una media, quindi, di 3 prescrizioni in due settimane. In particolare, vennero prescritti antibiotici a largo spettro (amoxicillina, amoxicillina+acido clavulanico e clindamicina) come unico trattamento, anche in assenza di coinvolgimento sistemico (febbre) e anche nel caso in cui la diagnosi fosse pulpite ${ }^{[7]}$.
L'abuso di antibiotici da parte degli odontoiatri secondo gli autori contribuisce nettamente all'antibiotico resistenza ${ }^{[5-7]}$. Riconoscere una situazione di "eccesso di prescrizioni" significa ammettere il ricorso al farmaco in condizioni in cui o non è indicato o non ha un ruolo primario nel trattamento di una data patologia. La conoscenza di regole precise e scientificamente validate rappresenta la condizione necessaria per non incorrere nel suddetto errore. Tra le patologie odontoiatriche per le quali più frequentemente viene fatto ricorso agli antibiotici annoveriamo le infezioni parodontali e peri-implantari.

La terapia della malattia parodontale è ormai assodato basarsi sulla rimozione del biofilm batterico, l'ottenimento di una superficie radicolare detossificata e adeguata a favorire i processi di guarigione seguita da un corretto mantenimento igienico.

Nonostante si tratti di una patologia a eziologia batterica, il metodo a oggi più efficace per rimuovere l'agente eziologico risulta il trattamento meccanico.

Tuttavia, la terapia meccanica non chirurgica in alcuni casi non riesce a eliminare tutti i microrganismi in tempi brevi, per cui la somministrazione di antibiotici sistemici e topici può essere vantaggiosa ${ }^{[8]}$.

In parodontologia due sono le modalità di somministrazione degli antibiotici: sistemica e topica.

L'efficacia clinica degli antibiotici somministrati per via sistemica nel trattamento della malattia parodontale è da decenni oggetto di valutazione e attualmente si è giunti a definirne le indicazioni. La somministrazione sistemica di antibiotici trova indicazione nelle forme acute e aggressive, secondo la definizione della classificazione dell'American Academy of Periodontology del 1999, ma sempre 
come un coadiuvante della terapia meccanica; risulta ingiustificata nel trattamento delle forme croniche ${ }^{[9,10]}$.

Anche per alcune forme acute, come l'ascesso parodontale, le attuali indicazioni suggerirebbero il ricorso all'antibiotico solo nei casi in cui la sintomatologia sia particolarmente severa e si tema per la diffusione della raccolta purulenta e per la salute generale del paziente ${ }^{[10,11]}$.

Deve essere comunque evidenziato che si tratta di forme la cui prevalenza si attesta attorno al $12 \%$ per quelle aggressive e al $14-25 \%$ per l'ascesso parodontale ${ }^{[12]}$. La scelta di un antibiotico sistemico da parte dei dentisti sembra spesso basarsi più su abitudini e consuetudini che non su evidenze scientifiche. Dati di vendita e studi evidenziano come i farmaci più prescritti siano i beta lattamici quali l'amoxicillina o l'amoxicillina in associazione con l'acido clavulanico[6]. Sebbene possano essere indicati nell'ascesso parodontale acuto risultano insufficienti nelle forme aggressive, dove è raccomandata l'associazione con metronidazolo o l'utilizzo di tetracicline.

La limitata efficacia clinica di tale modalità di somministrazione viene attribuita al fatto che solo minime quantità di farmaco raggiungono i microrganismi bersaglio, che sono collocati al di fuori dei tessuti parodontali e organizzati nel biofilm, che riduce marcatamente la sensibilità all'antibiotico stesso.

In quest'ottica da numerosi anni, i primi tentativi risalgono a Goodson et al. ${ }^{[13]}$, si sta lavorando all'impiego di antibiotici applicabili localmente che consentano di ottenere concentrazioni molto elevate di farmaco, mantenute per un tempo sufficientemente lungo, con un assorbimento sistemico praticamente nullo e quindi bassissimo rischio di effetti collaterali e di insorgenza di resistenze ${ }^{[8]}$.
I principi attivi più sperimentati sono le tetracicline (mino- e doxiciclina) e il metronidazolo, antibiotici propriamente detti nel cui spettro di azione rientrano i batteri parodontopatogeni, e antisettici come la clorexidina molto meno efficace anche in vitro.

Qualunque principio attivo ad azione antibiotica potrà avere proprietà curative della malattia umana, esercitando un'azione farmacologica sull'uomo, solo se in forma farmaceutica (Dir. 2001/83/CE) ${ }^{[14]}$. Quanto sopra, contraddistingue il "farmaco" ed è condizione necessaria per ottenere l'Autorizzazione all'Immissione in Commercio che è riportata sul confezionamento come numero a 9 cifre.

In generale, la forma farmaceutica che viene utilizzata per la somministrazione del principio attivo esercita una profonda influenza sulla velocità di assorbimento e sulla biodisponibilità del preparato farmacologico[15]. Nel caso specifico dei prodotti a uso locale, grande importanza riveste il carrier dal quale dipende la cinetica di rilascio. A tale proposito, si distingue in sistemi a rilascio controllato, che seguono una cinetica di "ordine 0 ") ${ }^{[16]}$, ossia un rilascio continuo del farmaco a una concentrazione costante e per un numero di giorni definiti, o rilascio lento dove la velocità di dismissione del farmaco è proporzionale alla quantità di prodotto residuo e tende quindi a diminuire nel tempo seguendo una cinetica di ordine primo $\left(1^{\circ}\right)$

Per quanto riguarda l'efficacia, studi clinici hanno dato risultati molto variabili riconoscendo a tali prodotti comunque utilità nel trattamento coadiuvante in tasche localizzate ma non come singolo trattamento. I risultati migliori venivano ottenuti con sistemi a rilascio controllato (fibre di tetraciclina, gel di doxiciclina iclato) che, a seguito di una singola applicazio- ne, garantivano concentrazioni efficaci per un periodo di almeno 12 gg $^{[16-18]}$.

L'efficacia di gel o di collutori a base di clorexidina nelle tasche parodontali si è dimostrata scarsa e comparabile al lavaggio con soluzione fisiologica ${ }^{[19]}$.

Un altro settore in cui vengono comunemente impiegati gli antibiotici in odontoiatria è quello delle patologie peri-implantari nelle forme di mucosite e peri-implantite.

Per il trattamento delle suddette patologie non esiste, al momento, un protocollo validato o linee guida definite. Nella grande varietà di alternative proposte, il ricorso all'uso dell'antibiotico sistemico è frequente sia in fase acuta che come coadiuvante durante il trattamento meccanico. Da un recente sondaggio, condotto tra parodontologi negli Stati Uniti, emerge che la maggior parte dei partecipanti ha dichiarato l'uso di antibiotici sistemici in combinazione alla terapia causale in presenza di peri-implantiti. L'antibiotico più somministrato è stata l'amoxicillina $(59,4 \%)$. Dai dati riportati si vede che I'antibiotico sistemico è ancora più utilizzato di quello topico[20]

A seguito di quanto sopra, appare evidente l'esistenza di un problema globale di insorgenza di resistenze batteriche agli antibiotici conseguente all'ampia diffusione dell'uso degli stessi. II settore odontoiatrico sembra contribuire in modo significativo in termini percentuali con il sospetto che vi sia un uso improprio o non coerente con le indicazioni date dalla letteratura

Il fine del lavoro è descrivere le modalità di prescrizione di antibiotici nel trattamento delle patologie parodontali e periimplantari coerentemente con le indicazioni scientifiche in un gruppo di dentisti italiani e discutere il problema dell'antibiotico resistenza. 


\section{MATERIALI E METOD|}

Lo studio consiste nell'analisi dei dati di un'indagine preesistente condotta da Edra Lswr Market Research, per conto di Hereus-Kulzer (azienda produttrice di antibiotico a uso locale). Nel luglio 2015, il questionario dal titolo "Gli antibiotici topici nel trattamento delle parodontiti e periimplantiti: quali indicazioni?" veniva proposto, senza criteri di selezione, ai 15.482 membri della popolazione di odontoiatri facenti parte della community certificata di Medikey di Edra. A distanza di alcuni mesi, veniva effettuato un secondo invio esclusivamente a coloro che non avevano ancora aderito evitando quindi ripetizioni.

Accettando l'invito, il partecipante veniva indirizzato tramite apposito link a una piattaforma on-line dove veniva presentata una serie di 13 domande chiuse con risposte a scelta multipla; a seconda dei quesiti, le alternative variavano da 3 a 5 e, ove indicato, potevano essere ammesse anche più preferenze. Per la gestione informatica ci si avvaleva della metodologia CAWI (Computer Aided Web Interview) che consente estrema rapidità nella raccolta dei dati e controlli sulla corretta compilazione da parte del rispondente.

Al fine di ottenere risposte focalizzate, nella lettera d'invito si specificava chiaramente che il sondaggio verteva esclusivamente sull'uso terapeutico degli antibiotici limitatamente alla parodontologia e all'implantologia.

Partendo dal dato di una popolazione di studi dentistici di 32.899 unità alla data del primo invio ${ }^{[1]}$, ponendo un intervallo di errore al $3 \%$ e un livello di confidenza al $95 \%$, la dimensione significativa del campione veniva calcolata in 1034 risposte. Nessun tipo di successiva inferenza statistica era stata presa in considerazione dagli ideatori del sondaggio.
Gli autori hanno curato esclusivamente l'interpretazione dei risultati, utilizzando la totalità del materiale disponibile, ma non hanno avuto ruolo nella preparazione del questionario.

Le domande presenti nel questionario erano le seguenti:

1. Con che frequenza prescrivi antibiotici sistemici?

2. Prescrivi antibiotico sistemico in caso di ascesso parodontale?

3. Come tratti una situazione di ascesso parodontale acuto?

4. Prescrivi antibiotico sistemico in caso di peri-implantite?

5. Potendo valutare l'incidenza di mucosite/peri-implantite nel tuo studio, cosa ti aspetteresti?

6. Sei al corrente dell'elevato numero di batteri patogeni resistenti all'amoxicillina?

7. Quanto sarebbe utile nella tua pratica clinica avere a disposizione un antibiotico topico?

8. Quali presidii professionali aggiuntivi utilizzi nella pratica professionale?

9. Hai mai usato antibiotici topici? Quali?
10. Con che frequenza usi gli antibiotici topici?

11. Quando hai usato l'antibiotico topico?

12. Conosci la differenza fra farmaco topico e medical device?

13. Conosci la differenza fra sistemi a rilascio controllato o lento?

\section{RISULTATI}

A fronte delle 15.482 mail del primo invio, si ottenevano 2868 (18,5\%) letture che generavano 633 (22\%) "click indagine" o risposte utilizzabili. II secondo invio, che escludeva coloro che avevano già risposto al primo mailing, si basava su 14.849 inoltri da cui $2694(18,1 \%)$ letture e 446 $(16,6 \%)$ risposte utili. Complessivamente quindi, hanno completato correttamente il sondaggio 1079 dentisti, ossia il 6,8\% del campione. La distribuzione territoriale dei professionisti che componevano la mailing list così come delle risposte (\%) è descritta nel grafico 1, in cui appare come, pur con pesi diversi, tutte le regioni risultino rappresentate.



Grafico 1 Composizione, su base geografica, del campione e delle risposte 
Ai fini del presente lavoro le risposte alle domande verranno presentate raggruppate in modo schematico per omogeneità di argomento e non rispettando la sequenza proposta. Si suddividerà quindi in risposte relative all'impiego degli antibiotici per via sistemica e successivamente locale.

\section{Impiego degli antibiotici per via sistemica}

\section{Con che frequenza prescrivi antibiotici sistemici?}

Per questa domanda le possibili indicazioni previste erano 3 con i seguenti risultati: il $44 \%$ delle risposte era per una prescrizione settimanale, il $31 \%$ per una somministrazione mensile e il rimanente $25 \%$ almeno una volta all'anno (grafico 2).

\section{Prescrivi antibiotico sistemico in caso di ascesso parodontale?} Questa domanda si apriva a due diverse possibilità; infatti, in caso di risposta affermativa veniva richiesto di esprimere anche la preferenza su alcuni farmaci di uso più comune. La prima informazione è che le risposte affermative erano il

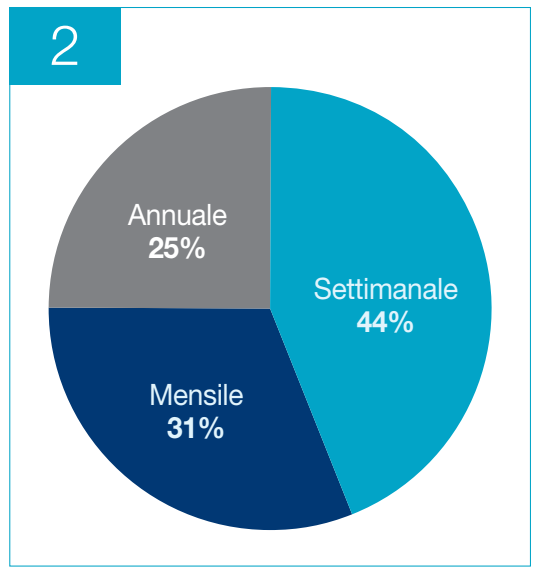

Grafico 2 Domanda 1: Con che frequenza prescrivi antibiotici sistemici?
91,6\%. Le preferenze prescrittive si distribuivano nel seguente modo: amoxicillina $10,6 \%$, amoxicillina+acido clavulanico $68,2 \%$, doxiciclina $1,6 \%$, metronidazolo $2,5 \%$, amoxicillina+metronidazolo $17,1 \%$ (grafico 3 ).

\section{Come tratti una situazione di ascesso parodontale acuto?}

Tra le alternative terapeutiche proposte le preferenze si distribuivano nel seguente modo: antibiotico sistemico 13,9\%, antibiotico sistemico+igiene orale $53 \%$, igiene professionale $1,9 \%$, igiene professionale e antibiotico topico $0,9 \%$, drenaggio e lavaggio con clorexidina $14,7 \%$, nessuna delle precedenti 2,7\% (grafico 4).

\section{Prescrivi antibiotico sistemico in caso di peri-implantite?}

Come per la domanda 2, anche in questo caso si aprivano due diverse possibilità a seconda che vi fosse risposta affermativa o meno. Le risposte positive risultavano il $79 \%$ con la seguente distribuzione per quanto riguarda le molecole utilizzate: amoxicillina $79 \%$, amoxicillina+acido clavulanico $62,7 \%$, doxiciclina $2,7 \%$, metronidazolo 2,5\%, amoxicillina+metronidazolo $22,9 \%$ (grafico 5 ).
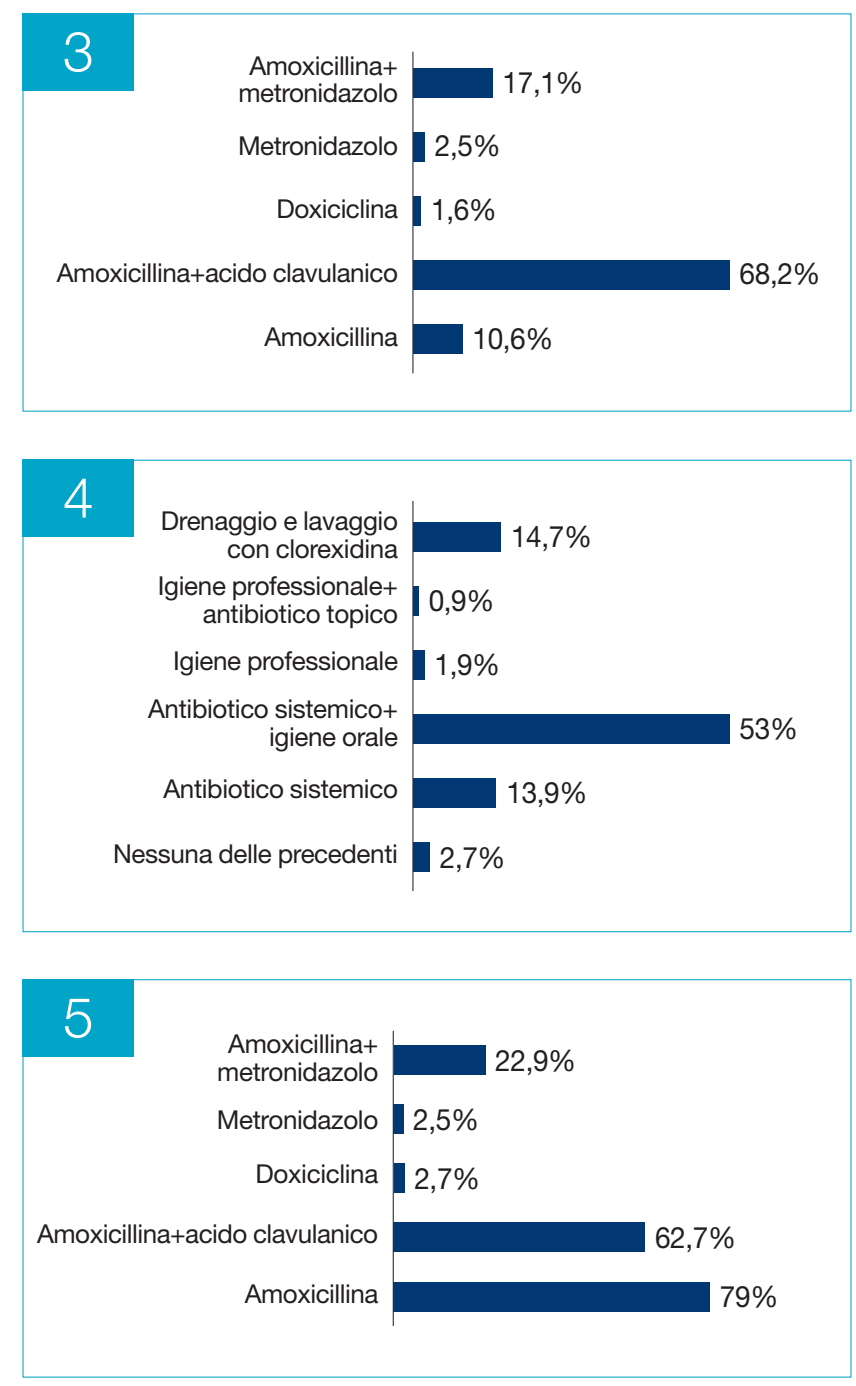

Grafico 3

Domanda 2: Prescrivi antibiotico sistemico in caso di ascesso parodontale?

Grafico 4 Domanda 3 : Come tratti una situazione di ascesso parodontale acuto?

Grafico 5 Domanda 4: Prescrivi antibiotico sistemico in caso di peri-implantite? 
5. Potendo valutare l'incidenza di mucosite/peri-implantite nel tuo studio, cosa ti aspetteresti?

Nel quesito vengono proposti 5 diversi intervalli così rappresentati: $0 \quad(3 \%)$, 0-10\% (66\%), 10-30\% (26\%), 30-50\% (4\%) e $>50 \%$ (1\%).

\section{Sei al corrente dell'elevato} numero di batteri patogeni resistenti all'amoxicillina?

In questo caso vi era solo l'alternativa tra affermazione e negazione e l'86\% delle risposte è stata per il sì.

\section{Impiego degli antibiotici a uso topico}

7. Quanto sarebbe utile nella tua pratica clinica avere a disposizione un antibiotico topico?

A questa domanda i partecipanti hanno risposto: pochissimo nell' $1,4 \%$ dei casi, poco nell' $8,9 \%$ dei casi, abbastanza nel $51,5 \%$ dei casi e molto nel $38,2 \%$ dei casi (grafico 6).

\section{Quali presidi professionali} aggiuntivi utilizzi nella pratica professionale?

Laser $28 \%$, fotodinamica $2 \%$, antibiotici topici $22 \%$, antisettici topici $46 \%$ e ozono $2 \%$ (grafico 7 ).

\section{Hai mai usato antibiotici topici?} Quali?

I| 97,3\% delle risposte confermava precedenti esperienze d'uso dell'antibiotico locale. Nello stesso quesito veniva chiesta anche la preferenza d'uso tra alcuni prodotti con i seguenti risultati: clorexidina gel $(31,1 \%)$, metronidazolo $(33,5 \%)$, minociclina $(6,5 \%)$, piperacillina $(11,2 \%)$, doxiciclina $8,5 \% \quad(9,2 \%)$ e doxiciclina $14 \%$ (8,5\%) (grafico 8).

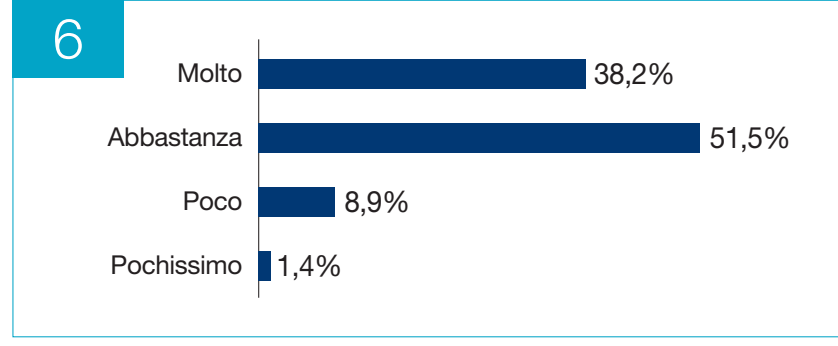

Grafico 6 Domanda 7: Quanto sarebbe utile nella tua pratica clinica avere a disposizione un antibiotico topico?

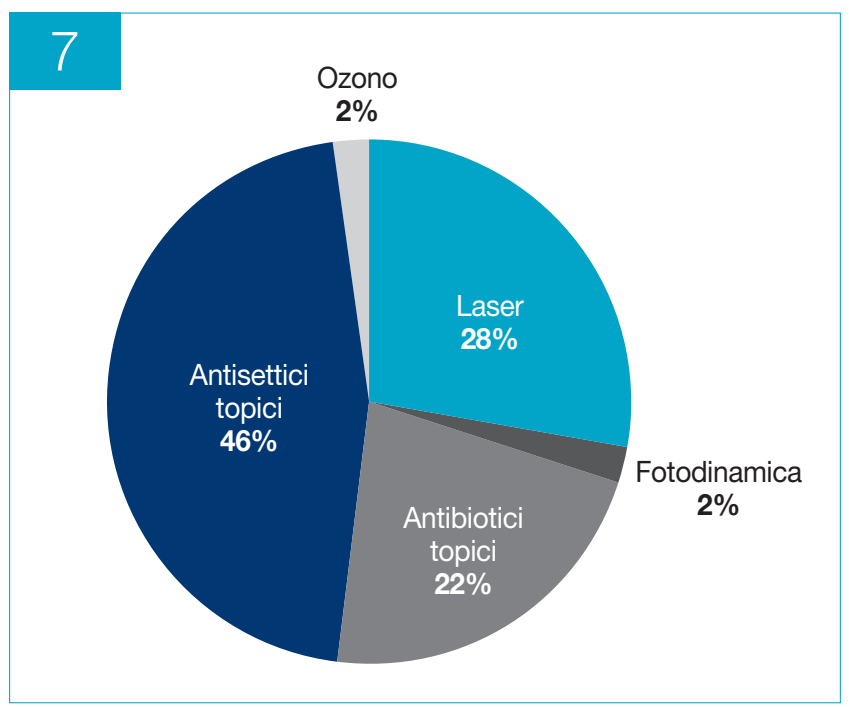

Grafico 7

Domanda 8:

Quali presidi professionali aggiuntivi utilizzi nella pratica professionale?

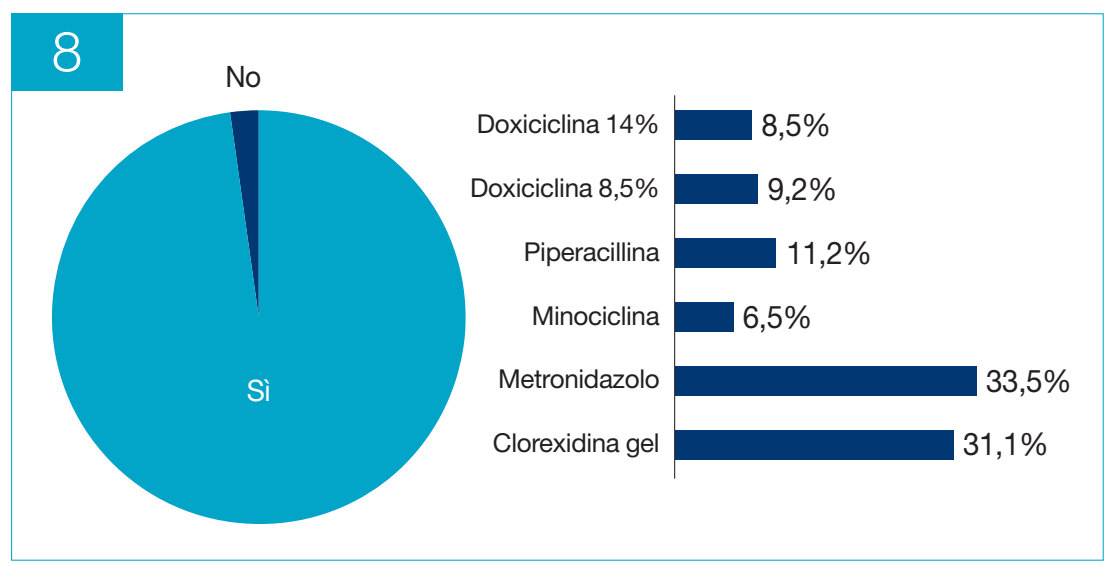

Grafico 8 Domanda 9: Ha mai usato antibiotici topici? Quali?

\section{Con che frequenza usi gli} antibiotici topici?

Valutando la frequenza d'impiego si è registrato che il $20,5 \%$ dei clinici lo usa almeno 1/alla settimana, il $22,7 \%$ almeno $1 / \mathrm{al}$ mese e il rimanente $56,8 \%$ qualche volta all'anno (grafico 9).

\section{Quando hai usato l'antibiotico topico?}

II questionario proponeva dei quadri clinici per cui potesse essere indicato l'antibiotico locale dando la possibilità di scegliere tra più opzioni. In questo modo vanno interpretati valori percentuali che 


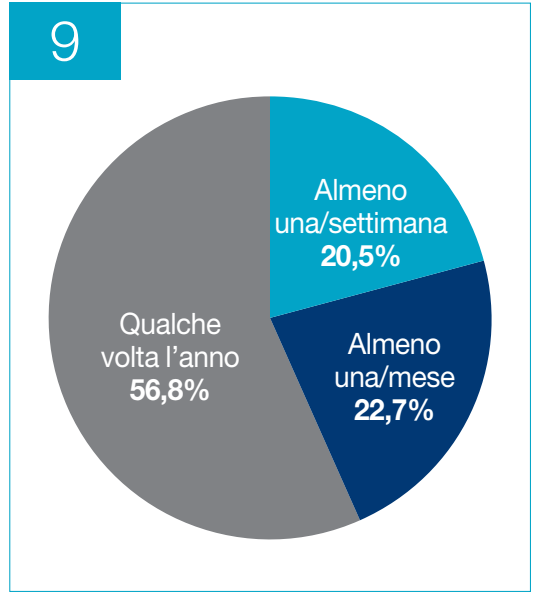

Grafico 9 Domanda 10: Con che frequenza usi gli antibiotici topici?

se sommati tra loro danno valore ben superiore al 100\%; in dettaglio, l'antibiotico locale veniva utilizzato dai dentisti del campione nel $31,7 \%$ dei casi di ascesso parodontale, nel $44,5 \%$ dei casi di periimplantite, nel $61,8 \%$ dei casi di tasche parodontali $>5 \mathrm{~mm}$, nel $20,4 \%$ dei casi di tasche residue e nel $49,9 \%$ dopo terapia non chirurgica nelle tasche profonde (grafico 10).

\section{Conosci la differenza fra} farmaco topico e medical device? 13. Conosci la differenza fra sistemi a rilascio controllato o lento?

La differenza tra farmaco topico e medical device risulta essere nota in meno della metà (43\%) dei partecipanti; più alta, $69 \%$, la porzione di coloro che conoscono la differenza tra le modalità di rilascio tra farmaci topici.

\section{DISCUSSIONE E INTERPRETAZIONE DEI RISULTATI}

La presente survey si basa su un numero di 1079 risposte che rappresentano il $6,8 \%$ dei possibili contatti e il $3,2 \%$ della popolazione odontoiatrica censita alla data dello studio ${ }^{[21]}$. Tale numero risulta in ogni caso adeguato sulla base del calcolo iniziale del sample size (1034). L'eventuale ragione tecnica (problemi d'invio, indirizzario non aggiornato o corretto, intervento di filtri ecc.) per l'evidente differenza tra "mail inviate" e "mail lette" non è nota agli autori. II grafico 1 evidenzia come il campione includesse dentisti di ogni regione d'Italia e come, similmente, le risposte utili provenissero dall'intero territorio nazionale; si osserva un evidente picco per la Lombardia mentre per la Val D'Aosta non si raggiunge l'unità percentuale.

La prima osservazione riguarda la frequenza di somministrazione per via sistemica: dalla domanda 1 si evince che il $44 \%$ dei rispondenti effettua una prescrizione alla settimana e complessivamente oltre il $70 \%$ almeno una volta al mese.

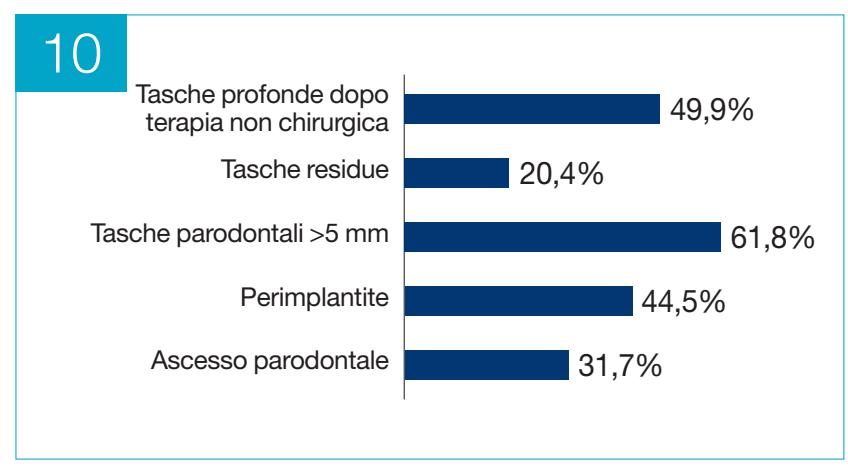

Grafico 10 Domanda 11: Quando ricorri all'uso dell'antibiotico topico?
Se si tiene conto delle premesse al questionario, ossia l'uso dell'antibiotico a solo scopo terapeutico, e che i quadri clinici per i quali ne è indicato l'impiego hanno una prevalenza relativamente bassa (ascesso parodontale: 12\%; forme aggressive: $14-25 \%)$ tale abitudine prescrittiva appare eccessiva ${ }^{[12]}$.

In effetti, il punto debole sembrerebbe essere proprio la conoscenza delle indicazioni cliniche. Le domande 2 e 3, relative al trattamento dell'ascesso parodontale, dimostrano il ricorso all'uso dell'antibiotico sistemico in circa il $92 \%$ dei casi e, addirittura, come unico trattamento nel $16 \%$. Le raccomandazioni scientifiche, qualora sia necessario il ricorso all'antibiotico, individuano l'amoxicillina come mono terapia o nelle forme più gravi in associazione all'acido clavulanico, l'antibiotico di prima di scelta ${ }^{[11,12]}$. I dati emersi rivelano però una maggior propensione all'uso di amoxicillina con acido clavulanico $(68,2 \%)$ addirittura ulteriormente associato a metronidazolo $(17,1 \%)$.

Con riferimento al trattamento della periimplantite (domanda 4) il ricorso all'uso di antibiotico sistemico avviene nel $79 \%$ dei casi. In realtà a differenza di quanto visto per alcuni quadri clinici parodontali, non esisterebbe in letteratura indicazione precisa all'uso di antibiotico ${ }^{[22]}$.

Analizzando i farmaci impiegati, si evidenzia una situazione analoga a quella descritta per l'ascesso parodontale con prevalente ricorso all'amoxicillina singolarmente o in associazione.

Un così diffuso utilizzo della suddetta molecola sembra stridere con i risultati della domanda 6 in cui l' $86 \%$ dei rispondenti si dichiara a conoscenza dell'elevato numero di batteri patogeni che hanno sviluppato resistenza all'amoxicillina stessa. 
Nel questionario si trovavano anche una serie di domande relative all'impiego di antibiotici locali. L'ampio interesse e il frequente ricorso all'utilizzo di tali prodotti viene ritrovato in varie risposte; nella domanda 8 , gli antibiotici topici $(22 \%)$ assieme agli antisettici topici (46\%) rientrano tra i più comuni "presidi professionali aggiuntivi". Alla possibilità di disporre di un antibiotico locale efficace viene attribuita un'importanza significativa in oltre l'86\% dei casi con "un grado di importanza" che variava da abbastanza $(51,5 \%)$ a molto (38\%) (domanda 7$)$. A ulteriore conferma, non solo la quasi totalità dei rispondenti $(97,3 \%)$ (domanda 9) ha utilizzato almeno una volta antibiotici locali, ma quasi la metà $(43,2 \%)$ continua a usarli con una frequenza mensile o più (20,5\% una volta alla settimana e $22,7 \%$ una volta al mese) (domanda 10).

Nella domanda 11, si chiedeva in quali circostanze venissero utilizzati gli antibiotici locali suggerendo alcuni quadri clinici. I risultati evidenziavano come tutte le situazioni proposte rientrassero ef- fettivamente tra le scelte dei clinici pur con un maggiore ricorso sia nella periimplantite che nelle situazioni con tasche parodontali profonde. Un così ampio e vario impiego conferma la sensazione che l'antibiotico locale venga considerato un importante aiuto nella terapia dei quadri più impegnativi, anche se talvolta senza un reale supporto scientifico alla scelta.

Essendo assodato che l'efficacia di un antibiotico a somministrazione locale è legata alla formulazione farmaceutica (veicolo) da cui dipende la farmacocinetica, nella survey si è voluto sondare il grado di conoscenza di tale aspetto. Oggetto della domanda 13 era la differenza fra i diversi sistemi di rilascio (controllato vs lento): la percentuale di risposte positive non raggiunge il $70 \%$ ed è probabilmente sovrastimata perché la modalità con cui viene posta la domanda tende a suggerire o a condizionare la risposta in senso positivo. Lo stesso si può dire quando si valuta la risposta alla domanda 12 dove le risposte positive a riguardo della differenza tra "medical device" e "farmaco" si attestano attorno al 43\%. Tali valori dimostrano la mancata conoscenza, da parte di un'ampia percentuale dei clinici intervistati, di alcuni dei criteri fondamentali per la scelta del prodotto più adeguato.

Tali carenze possono aiutare a interpretare il risultato della domanda 9 nella parte relativa al tipo di prodotto utilizzato che vede la clorexidina gel impiegata nel $31 \%$ dei casi. Tale prodotto non è un antibiotico, nel suo spettro di azione non rientrano molti dei batteri parodonto-patogeni e, al di là della sostantività propria della molecola, il gel non può garantire alcuna delle caratteristiche richieste per un farmaco a uso locale. Ricordiamo, infatti, che la clearance del fluido crevicolare elimina in 12,5 minuti qualsiasi tipo di gel che non abbia caratteristiche di rilascio controllato ${ }^{[23]}$. L'uso estensivo può quindi essere attribuibile a ragioni di economicità e di facile reperibilità, forse visti come criteri più importanti rispetto all'efficacia clinica.

\section{CONCLUSIONI}

I dati analizzati, pur nei limiti dello studio e delle dimensioni del campione peraltro rappresentativo, sembrano indicare che il ricorso all'uso di antibiotici per via sistemica, anche in associazione, sia frequente ed eccessivo se confrontato con i valori di prevalenza dei relativi quadri patologici[i2].

Tale atteggiamento sembrerebbe non essere coerente con le indicazioni scientifiche e con l'esigenza di cercare di arginare il problema dell'insorgenza di resistenze batteriche ${ }^{[24]}$.

Il ricorso alle formulazioni locali risulta essere molto apprezzato, ma la scelta del prodotto utilizzato non sembrerebbe essere sempre guidata da un'adeguata conoscenza scientifica, ma piuttosto subordinata ad altri criteri quali l'economicità. 


\section{BIBLIOGRAFIA}

1. La Placa M. Principi di microbiologia medica. $13^{a}$ Edizione. Bologna: Società Editrice Esculapio, luglio 2012;8:184-5.

2. ECDC. Antimicrobial resistance surveillance in Europe. Annual report of the European Antimicrobial Resistance Surveillance Network (EARS-Net), 2014.

3. Marra F, George D, Chong M, Sutherland S, Patrick DM. Antibiotic prescribing by dentists has increased. Why? JADA 2016;147(5): 320-7.

4. Pipalova R, Vlcek J, Slezak R. The trends in antibiotic use by ge neral dental practitioners in the Czech Republic (2006-2012). International Dental Journal 2014;64:138-43.

5. Karki AJ, Holyfield G, Thomas D. Dental prescribing in Wales and associated public health issues. British Dental Journal 2001;210(1):E21.

6. Poveda-Roda R, Bagán JV, Sanchis-Bielsa JM, Carbonell-Pastor E. Antibiotic use in dental practice. A review. Med Oral Patol Oral Cir Bucal 2007;12:E186-92.

7. Mainjot A, D'Hoore W, Vanheusden A, Van Nieuwenhuysen J-P. Antibiotic prescribing in dental practice in Belgium. International Endodontic Journal 2009;42:1112-7.

8. Mombelli A, Lakshman P. Samaranayake. Topical and systemic antibiotics in the management of periodontal diseases. International Dental Journal 2004;54:3-14.

9. Armitage GC. Development of a classification system for periodontal diseases and conditions. Ann Periodontol 1999 Dec;4(1):1-6.

10. Albandar JM. Aggressive and acute periodontal diseases. Periodontology 2000 2014;65:7-12.

11. Dahlen G. Microbiology and treatment of dental abscesses and periodontal-endodontic lesion. Periodontology 2000 2002;28:206-39.

12. Herrera $\mathbf{D}$, Roldàn $\mathbf{S}$, Sanz $\mathbf{M}$. The periodontal abscess: a review. J Clin Periodontol 2000;27(6):377-86.

13. Goodson JM, Haffajee A, Socransky SS. Periodontal therapy by local delivery of tetracycline. Journal of Clinical Periodontology 1979:6:83-92
14. Directive 2001/83/EC of the European Parliament and of the council of 6 november 2001 on the community code relating to medicinal products for human use. Official Journal L - 311, 28/11/2004: 67-128. 15. AIFA. Glossario del Farmaco, www.AIFA.gov.it.

16. Tonetti M, Cugini MA, Goodson JM. Zero-order delivery with periodontal placement of tetracycline-loaded ethylene vinyl acetate fibers. J Periodontal Res 1990;25(4):243-9.

17. Tonetti MS, Lang NP, Cortellini $P$, Suvan JE, Eickholz $P$, Fourmousis I et al. Effects of a single topical doxycycline administration adjunctive to mechanical debridement in patients with persistent/ recurrent periodontitis but acceptable oral hygiene during supportive periodontal therapy. J Clin Periodontol 2012;39:475-82.

18. Ratka-Krüger P, Schacher B, Bürklin T, Böddinghaus B, Holle R, Renggli $\mathrm{HH}$ et al. Non-surgical periodontal therapy with adjunctive topical doxycycline: a double-masked, randomized, controlled multicenter study. II. Microbiological results. Journal of Periodontology 2005;76(1):66-74

19. Wennström JL, Heijl L, Dahlén G, Gröndahl K. Periodic subgingival antimicrobial irrigation of periodontal pockets. J Clinical Periodont 1987; $14: 541-50$

20. Papathanasiou E, Finkelman M, Hanley J, Parashis AO. Prevalence, etiology and treatment of peri-implant mucositis and peri-implantitis: a survey of periodontists in the United States. J Periodontol 2016 Maj;87(5):493-501.

21. Dental Directory - Annuario Dentale, $29^{\mathrm{a}}$ edizione, 2015. Peschiera Borromeo (MI): O.VE.S.T. srl.

22. Dahlen G. Microbiology and treatment of dental abscesses and periodontal-endodontic lesion. Periodontology 2000 2002;28:206-39.

23. van Winkelhoff AJ. Antibiotics in the treatment of peri-implantitis. Eur J Oral Implantol 2012;5 Suppl: S43-50.

24. Mensi M, Scotti E, Francetti L, Giargia M, Latronico M et al. Passato, presente e futuro dei coadiuvanti locali e sistemici nel trattamento non chirurgico di perimplantiti e parodontiti: indicazioni e limiti. Dental Cadmos 2016;84(3):2-15. 


\section{Long Abstract}

\section{INTRODUCTION}

The discovery of antibiotics and the subsequent synthesis of new molecules represent an important step in medicine.

The implementation of new systemic pharmaceutical formulations and the manageability of drugs have further spread its use and benefits. However, due to the widespread diffusion, the problem of the emergence of bacterial resistance to the drugs followed by the loss or decrease of efficacy and a resurgence of pathologies. In periodontology there are two methods of antibiotic administration: systemic and topical. Aim of the present study was to evaluate the use of systemically and locally delivered antibiotics for the treatment of periodontal end peri-implant diseases in a sample of italian dentists. Furthermore the accordance to evidence based indications and the issues of antibiotic resistance were examined.

\section{MATERIALS AND METHODS}

The study consists in the analysis of the data of a pre-existing survey conducted by Edra Lswr Market Research, on behalf of Hereus-Kulzer. A questionnaire entitled "Topical antibiotics in the treatment of periodontitis and peri-implantitis: what indications?" was proposed, without selection criteria, to the 15,482 members of the population of dentists belonging to the certified community of Medikey of Edra.

The authors of the study took care of the interpretation of the results obtained.

\section{RESULTS}

The results of the present survey is based on a number of 1079 responses representing $6.8 \%$ of the possible contacts and $3.2 \%$ of the dental population at the time of the study. The results showed that the use of systemic antibiotics for periodontal and peri-implant disease was highly frequent. Amoxicillin in association to clavulanic acid were the most prescribed therapy. The use of local antibiotics including clorexydine was also very frequently proposed.

\section{CONCLUSIONS}

Systemic administration of antibiotics appears as the first choice of treatment also when not strictly indicated. Within the limits of this study, the data analyzed seem to confirm that the use of antibiotics by systemic route is frequent and excessive if compared with respect the prevalence values of related pathological findings. This attitude seems not to be consistent with the scientific indications and with the need to try to stem the problem of the onset of bacterial resistance. Locally delivered antibiotics and antiseptic are also very frequently used in conjunction with different altered clinical conditions. However, the use of local formulations is very appreciated but the choice of the product used does not seem to be always guided by an adequate scientific knowledge but rather subordinated to other criteria such as economy of the products.

\section{CLINICAL SIGNIFICANCE}

The widespread diffusion and use of antibiotics and the related problem of the emergence of bacterial resistance to antibiotics followed by loss or decrease of efficacy with the consequent resurgence of pathologies is a contemporary problem. According to the results of the present study, in periodontology systemic administration of antibiotics appears as the first choice treatment also when not strictly indicated. Within the limits of this study, the data analyzed seem, in fact, to confirm that the use of antibiotics by systemic route is frequent and excessive if compared with respect the prevalence values of related pathological findings. 